\title{
Tailoring NIR-II photoluminescence of single thiolated Au25 nanoclusters by selective binding to proteins
}

Franck Bertorelle

ILM CNRS and Univ Lyon1

David Wegner

Division Biophotonics (BAM-1.2) Federal Institute for Materials Research and Testing (BAM)

Martina Perić Bakulić

University of Split https://orcid.org/0000-0002-9134-5500

Hussein Fakhouri

ILM

Clothilde Comby-Zerbino

ILM

\section{Amin Sagar}

Institute of Microbial Technology https://orcid.org/0000-0002-6207-9557

\section{Pau Bernadó}

CNRS

\section{Ute Resch-Genger}

Federal Institute for Materials Research and Testing https://orcid.org/0000-0002-0944-1115

\section{Vlasta Bonačić Koutecky}

Chemistry Department, Humboldt University of Berlin

\section{Xavier Le Guével}

Grenoble Alpes University

Rodolphe Antoine ( $\nabla$ rodolphe.antoine@univ-lyon1.fr)

ILM CNRS and Univ Lyon1 https://orcid.org/0000-0001-5682-8550

\section{Article}

Keywords: gold nanoclusters, small-angle X-ray scattering, molecular modeling

Posted Date: October 21st, 2021

DOI: https://doi.org/10.21203/rs.3.rs-958149/v1 
License: (c) (i) This work is licensed under a Creative Commons Attribution 4.0 International License. Read Full License 


\section{Abstract}

Atomically precise gold nanoclusters (Au NCs) are a fascinating class of nanomaterials that exhibit molecule-like properties and have outstanding photoluminescence $(\mathrm{PL})$, which is highly dependent on their structure and chemical environment. Their ultrasmall size, molecular chemistry, and biocompatibility make them extremely appealing for selective biomolecule labeling in investigations of biological mechanisms at the cellular and anatomical levels. In this work, we report a simple route to incorporating a preformed Au25 nanocluster into a model bovine serum albumin (BSA) protein. A new approach combining small-angle $X$-ray scattering and molecular modeling provides a clear localization of a single Au25 within the protein to a cysteine residue on the gold nanocluster surface. Attaching $\mathrm{Au}_{25}$ to BSA strikingly modifies the PL properties with enhancement and a redshift in the second near-infrared window (NIR-II). An extensive study based on a bottom-up approach that uses mixed-ligand nanoclusters $\mathrm{Au}_{25} \mathrm{pMBA}_{(18-\mathrm{x})} \mathrm{Cys}_{\mathrm{x}}$ with $\mathrm{x}=2,5,18$ supported by experimental data (steady state, time-resolved spectroscopy) and theoretical calculations (DFT) provides new hints at the origin of NIR-II emission in such nanoclusters and their subsequent enhancement when selectively binding to a cysteine-rich protein. This study paves the way to controlling the design of selectively sensitive probes in biomolecules through a ligand-based strategy to enable the optical detection of biomolecules in a cellular environment by live imaging.

\section{Introduction}

Gold nanoclusters (Au NCs) are at the forefront of nanomaterials that can be used for bio-applications due to their high colloidal stability, ultra-small size, low toxicity, high biocompatibility, and size-tunable photoluminescence $(\mathrm{PL}) .1,2,3,4,5$ The ability to tailor their surface offers great advantages to label (bio)molecules of interest and monitor them using advanced imaging techniques. ${ }^{6}$ However, Au NCs possess intrinsically quite low quantum yields (QYs); thus, several rational strategies have been developed to tailor and increase their PL properties. ${ }^{7,8}$ For example, one strategy involves increasing the organic shell rigidity, which protects the metal core and has led to several-fold PL enhancement in the near-infrared NIR-I (700-900 nm) $)^{9,10,11}$ and NIR-II (900-1700 nm) ${ }^{12}$ regions. This PL increase has been associated with the reduction of water molecules in the proximity of the Au NC surface, which minimizes non-radiative recombination processes. ${ }^{13} \mathrm{~A}$ second strategy relies on the fine tuning of the surface chemistry. ${ }^{14}$ Millstone's team conducted an extensive study demonstrating the influence of thiolated ligands ${ }^{15}$ on the near infrared emission of ultrasmall metal (gold, silver, copper) nanoparticles. ${ }^{16,17}$ In addition, it was shown that the addition of a thiolated ligand is important, and the denticity of the ligand used can influence the PL band and QY. For example, adding a short dithiol co-ligand to the Au NC surface led to a 6-fold increase in the QY with PL enhancement in the 1100-1300 $\mathrm{nm}^{18}$ range, which allowed the researchers to monitor them noninvasively in vivo down to a $4 \mathrm{~mm}$ depth in mice. ${ }^{19}$

Protecting Au NCs within large biomolecules such as proteins can be a rational and elegant strategy for engineering the PL of Au NCs and lead to ideal platforms for biomedical applications. ${ }^{20}$ The presence of 
intrinsic reducing agents and sulfur-containing chemical groups in proteins enables the one-step protection of metal NCs. The synthesis of fluorescent protein-directed Au NCs using albumin (BSA, bovine serum albumin) as both a reductant and stabilizer was pioneered by Xie et al. ${ }^{21}$ Following this seminal work, various NCs were synthesized with different proteins, showing very high PL brightness and good photostability for biolabeling/imaging purposes.. ${ }^{22,23,24}$ Although some studies have shown that proteins retain their structure and biological activity upon protein-directed NC formation, ${ }^{25,26,27}$ there is an increasing number of studies demonstrating that the global (and local) structure of proteins is altered after NC formation; these studies were often performed under quite harsh conditions ( $\mathrm{pH} 12$, for example), which can also affect the structure of the proteins. ${ }^{28,29,30,31,32}$

Additionally, the exact number of metal atoms in protein-directed NCs and their localization within the protein are still under debate, preventing the development of a clear structure-property relationship. ${ }^{7}$ It has been recently shown that clusters in proteins may in fact be composed of sub-units of 8 to 10 atoms located in different parts of the protein, and these sub-units can induce partial denaturation. ${ }^{33,34}$ Clearly, with such alteration of the native state of the protein host and/or uncontrolled formation and location of clusters within proteins, such nanoclusters may present reduced bioactivity and/or biocompatibility, limiting the widespread use of such materials in biomedical research. Therefore, new synthetic methods preserving the structure and bioactivity of incorporated NCs are in high demand. ${ }^{35}$ Ligand exchange plays an important role in thiolated nanoclusters, as the flexibility of the gold-sulfur interface enables post-synthetic modification. ${ }^{36}$ Additionally, functionalization of gold through ligand exchange has been recently exploited for protein carbonylation detection. ${ }^{37,38}$ As mentioned before, variations in the nature of ligands and the simplicity of the ligand exchange offer great potential to amplify specific properties of the clusters, such as the PL properties. ${ }^{7}$

In this work, we report for the first time the incorporation of a single preformed $\mathrm{Au}_{25}$ nanocluster $\left(\mathrm{Au}_{25}(\mathrm{pMBA})_{18}, \mathrm{pMBA}\right.$ - 4-mercaptobenzoic acid) into bovine serum albumin (BSA) protein selectively anchored to one cysteine residue without drastic modification of the protein structure. The attachment of a single thiolated $\mathrm{Au}_{25}$ nanocluster to the protein had a significant effect on its NIR-II emission band with a 4.7-fold enhancement of the PL QY. Such water-soluble $\mathrm{Au}_{25} \mathrm{NCs}$ attached to BSA display bright infrared emission between $700 \mathrm{~nm}$ and $1300 \mathrm{~nm}$, in contrast to previously reported fluorescent BSAdirected Au NCs that only emit below $1000 \mathrm{~nm}$. Mass spectrometry analysis revealed that the attachment of $\mathrm{Au}_{25} \mathrm{NCs}$ to BSA involves ligand exchange between the pMBA ligands and BSA cysteine (Cys) residues. Small-angle X-ray scattering (SAXS) provided an unambiguous localization of a $\mathrm{Au}_{25} \mathrm{NC}$ on the protein via a free cysteine residue, Cys34. To better understand the PL properties of $\mathrm{Au}_{25}$ nanoclusters attached to $B S A$, we synthesized mixed liganded nanoclusters, $A_{25} \mathrm{pMBA}_{(18-\mathrm{x})} \mathrm{Cy} \mathrm{s}_{\mathrm{x}}$ with $\mathrm{x}=2,5,18$, through ligand exchange. The origin of NIR-II emission in nanoclusters with mixed ligands determined by optical measurements and density functional theory reveals the key role of the metallic-ligand interface in photophysical pathways. These results pave the way for the tailored synthesis and protein bioconjugation of nanoclusters to extend their uses for diagnostic and therapeutic applications. 


\section{Results}

Formation of $\mathrm{Au}_{25} \mathrm{pMBA}_{18}$ nanoclusters and their attachment to $\mathrm{BSA}$. $\mathrm{Au}_{25}$ clusters protected by 18 mercaptobenzoic acid (para isomer) ligands were synthesized as described in a previous study. ${ }^{39}$ The best results were obtained when a slight excess of $\mathrm{Au}_{25} \mathrm{PMBA}_{18}$ over BSA (ratio 1.2:1) was used, but the synthesis was also performed with an excess of protein (ratio 1:3). Upon gentle and continuous stirring for 3 days, the $\mathrm{Au}_{25}$ nanocluster was incorporated into the BSA protein via ligand exchange between the sulfur groups from pMBA and the sulfur group from cysteine-containing amino acid residues in the BSA. A schematic representation of the proposed reaction mechanism is shown in Figure 1. These formed $\mathrm{Au}_{25}$-BSA conjugates were purified using $10 \%$ acrylamide SDS-PAGE gel separation. The gel resulted in two bands, which were attributed to $\mathrm{Au}_{25} \mathrm{PMBA}_{18}$ and $\mathrm{BSA}-\mathrm{Au}_{25}$, in contrast with the single band observed for the BSA control (Fig. S1). The slight difference in the positions of the BSA and BSA-Au 25 bands in the gel indicates that both the charge and overall shape of the protein subtly changed due to the incorporation of the preformed $\mathrm{Au}_{25} \mathrm{NCs}$ into the BSA protein. We noticed that $\mathrm{Au}_{25} \mathrm{pMBA}_{18}$ could not bind to BSA even when we used an excess of protein (Fig. S1). This result could suggest that $\mathrm{Au}_{25} \mathrm{pMBA}_{18}$ is not stuck to the surface but fixed inside the protein and cannot react anymore.

Characterization of $\mathrm{Au}_{25}$-BSA conjugates. Matrix-assisted laser desorption/ionization time-of-flight mass spectrometry (MALDI-TOF MS) was used to characterize the BSA-Au NCs (Figure 2a). In addition to the signature peak of the BSA protein at $66 \mathrm{kDa}$, the MALDI spectrum shows an additional peak at higher molecular weight. The molecular weight difference of this peak from BSA was 7.3 kDa, which indicates the attachment of only one gold nanocluster to the protein, as the mass for one $\mathrm{Au}_{25} \mathrm{pMBA}_{18}$ nanocluster is $7.68 \mathrm{kDa}$. However, the observed mass shift of the $\mathrm{Au}_{25}-\mathrm{BSA}$ was lower than expected. This discrepancy can be explained by the removal of 2-3 pMBA surface ligands during incorporation into BSA and the replacement of intrinsically available sulfur groups from the cysteine residues in the BSA. The first ligand exchange may preferentially occur with only the free cysteine residue of the BSA (Cys34), and then 1 or 2 cysteines in the disulfide bridge may become available to bind the $\mathrm{Au}_{25} \mathrm{NCs}$ in the BSA (see Fig. S2).

Secondary structural changes in the $\mathrm{BSA}$ upon $\mathrm{Au}_{25} \mathrm{NC}$ incorporation were measured by circular dichroism (CD) spectroscopy, which is shown in Figure S3. The CD spectra of the BSA and BSA-Au ${ }_{25}$ are very similar, suggesting that the $\mathrm{BSA}-\mathrm{Au}_{25}$ retained the a-helicity to a high degree. This observation also confirms the low perturbation of the protein secondary structure content. These results are in stark contrast to the results of Xie's classical method, which was performed under alkaline conditions, and they show the advantage of the synthetic methodology developed here. ${ }^{21}$

Small-angle X-ray scattering (SAXS) is a widely used technique for the structural and dynamic characterization of biomolecules in aqueous solutions. ${ }^{40,41,42}$ To obtain the scattering profiles of BSA and the BSA-Au ${ }_{25}$ complex, inline size-exclusion chromatography coupled to SAXS (SEC-SAXS) was used, as it enables the separation of the major peak corresponding to the BSA monomer from those of the BSA 
oligomers (Fig. S4a). Consistent with its larger molecular weight, BSA-Au ${ }_{25}$ eluted earlier than native BSA. SEC-SAXS frames corresponding to the monomer were averaged using standard procedures for subsequent structural analyses. The SAXS profiles of $\mathrm{BSA}$ and $\mathrm{BSA}-\mathrm{Au}_{25}$ present different features along the momentum transfer range measured, indicating that the presence of the $\mathrm{Au}_{25} \mathrm{NC}$ strongly modifies the scattering properties of the protein (Fig. $2 \mathrm{~b}$ ). As expected, $\mathrm{BSA}-\mathrm{Au}_{25}$ presents a slightly larger radius of gyration, $R_{g}$, than free BSA, $28.16 \pm 0.05 \AA$ and $27.95 \pm 0.02$ and, respectively. When computing the pairwise distance distribution, $p(r)$, we found that the unmodified BSA had a $D_{\max }$ of $82.1 \AA$, similar to the value identified in earlier studies ${ }^{43}$, and a symmetric $p(r)$ profile typical of globular proteins. In contrast, BSA- $\mathrm{Au}_{25}$ had a significantly larger $D_{\text {max }}$ of $90.85 \AA$ and had a shoulder at small distances, indicating the presence of additional scattering centers in the protein.

Next, the capacity of the crystallographic structure of BSA (PDB entry 3v03) was evaluated to describe both SAXS curves shown in Fig. 2b. While the structure was in excellent agreement with the BSA profile $\left(X^{2}=2.2\right)$ (Fig. 2b), unsurprisingly, it was unable the describe the curve measured for $B S A-A_{25}\left(X^{2}=24.6\right)$. To achieve a better fit between the experimental data and the theoretical structure of the $B S A-\mathrm{Au}_{25}$, we modeled the $\mathrm{Au}_{25} \mathrm{NC}$ (see Computational for $\mathrm{Au}_{25} \mathrm{NC}$ details in the $\mathrm{SI}$ ) on the surface of the BSA. we selected Cys 34 for this model, as it is the only free cysteine in the protein, and we placed its side chain in the position of one of the pMBA molecules of the external protective shell of the Au NC (Fig. 2c). Although the incorporation of the $\mathrm{Au}_{25} \mathrm{NC}$ into the BSA structure improved the agreement with the experimental $B S A-A_{25}$ SAXS curve $\left(X^{2}=6.55\right)$, systematic deviations throughout the momentum transfer range were still observed. A closer inspection of the BSA structure showed a region of the protein (residues 55-119), consisting of a long unstructured loop (residues 105-119), that could be structurally impacted in the presence of the NC. To test this hypothesis, we performed a normal mode SAXS refinement of the BSA$\mathrm{Au}_{25}$ structure using the program SREFLEX, ${ }^{44}$ allowing movement in this region while maintaining the rest of the protein and keeping the NC fixed in place (Fig. 2c).

To show the selectivity of $\mathrm{NC}$ binding, we generated seven different structures with the $\mathrm{Au}_{25} \mathrm{NC}$ attached to other cysteines of the BSA (Fig. 2d). Note that these cysteines are engaged in disulfide bonds in the native structure. For six of the $\mathrm{BSA}-\mathrm{Au}_{25}$ complexes, the resulting structures displayed worse $\chi^{2}$ values than the model built using Cys34 as the anchoring point (Fig. 2e). Even after normal mode refinement, all the resulting structures provide worse agreement with the experimental data than the Cys34-anchored structure. In short, the SAXS analyses strongly indicate that the $\mathrm{Au}_{25} \mathrm{NC}$ selectively binds to free Cys34 and that this binding induces structural perturbations to accommodate the metallic cluster.

Optical properties of the $\mathrm{Au}_{25} \mathrm{PMBA}_{18}$ and $\mathrm{BSA}-\mathrm{Au}_{25} \mathrm{NCs}$. Figure $3 \mathrm{a}$ shows the absorption spectra of $\mathrm{Au}_{25} \mathrm{PMBA}_{18}$ and $\mathrm{BSA}-\mathrm{Au}_{25} \mathrm{NCs}$ dispersed in water, with both clusters presenting the "fingerprint" band at $\sim 680 \mathrm{~nm}$, which is characteristic of $\left[\mathrm{Au}_{25}(\mathrm{SR})_{18}\right]^{-} \mathrm{NCs} .{ }^{45}$ The absorption spectra of $\mathrm{Au}_{25} \mathrm{pMBA}_{18}$ and $\mathrm{BSA}-\mathrm{Au}_{25}$ clusters are similar to the 25-Au-atom cluster $\left(\mathrm{Au}_{25}\left(\mathrm{SCH}_{2} \mathrm{CH}_{2} \mathrm{Ph}\right)_{18}\right)$ with a known crystallographic structure. ${ }^{46}$ This result suggests that they all have similar structural features, although 
UV-vis absorption is not the method of choice to determine or compare structures. The attachment of $\mathrm{Au}_{25}$ to $\mathrm{BSA}$ leads to a significant enhancement of the PL signal (upon excitation at $700 \mathrm{~nm}$ ) at both 850 $\mathrm{nm}$ and $1050 \mathrm{~nm}$, as seen in Figure 3b, with a 4.7-fold increase in QY (see Figure 3d). A similar trend is observed for excitation at $400 \mathrm{~nm}$ (see Fig. 3c). To confirm that this effect is not only due to simple physisorption of $\mathrm{Au}_{25} \mathrm{NCs}$ onto BSA, steady-state and time-resolved PL measurements (Figure $3 \mathrm{e}$ ) of $\mathrm{Au}_{25} \mathrm{pMBA}_{18}$ and $\mathrm{BSA}-\mathrm{Au}_{25}$ were performed in water, and $\mathrm{Au}_{25} \mathrm{pMBA}_{18}$ was analyzed in the presence of a high BSA concentration ( $50 \mathrm{mg} / \mathrm{mL}$ ). Although the addition of BSA resulted in a PL intensity enhancement, the overall effect was much lower than that of direct incorporation. Better insight into the influence of BSA on the fluorescence kinetics of Au NCs can be obtained by time-resolved measurements. The addition of BSA to the Au NC solution resulted in an increase in the average amplitude weighted lifetime from $72.1 \mathrm{~ns}$ to $396.3 \mathrm{~ns}$. However, the $\mathrm{Au}_{25}-\mathrm{BSA}$ complex possessed a PL lifetime of $936.1 \mathrm{~ns}$, which indicates that the local environment of the $\mathrm{Au}_{25} \mathrm{NCs}$ in $\mathrm{BSA}-\mathrm{Au}_{25}$ is more rigid than that of free $\mathrm{Au}_{25} \mathrm{pMBA}_{18}$ dispersed in water or in the presence of unbound protein. Therefore, the tight entrapment of $\mathrm{Au}_{25}$ in the protein scaffold of $\mathrm{BSA}-\mathrm{Au}_{25}$ is prone to multiple energy transfers associated with intersystem crossings (vide infra). ${ }^{47,48}$

The absence of any changes in the PL intensity and lifetime of the $\mathrm{Au}_{25}-\mathrm{BSA}$ complex when added to a concentrated BSA solution is additional evidence for the successful incorporation of the cluster in the BSA protein and thus protection from its environmental surroundings (Figure S5), indicating the presence of a strong protective shell around the $\mathrm{Au}_{25}$ in this labeled protein.

In summary, by attaching a single $\mathrm{Au}_{25} \mathrm{NC}$ to $\mathrm{BSA}$ through ligand exchange between protecting pMBA ligands and natural sulfur-containing BSA cysteine residues, we managed to tailor the NIR-II signal while keeping the structure of $\mathrm{Au}_{25}$ intact. Interestingly, the ratio between the relative intensity at $850 \mathrm{~nm}$ and $1050 \mathrm{~nm}$ strongly depends on the excitation wavelength (compare $\lambda_{\text {exc. }}=400 \mathrm{~nm}$ and $\lambda_{\text {exc. }}=700 \mathrm{~nm}$, see Fig. 3 and Table S1). The $\mathrm{I}_{1050} / \mathrm{l}_{850 \mathrm{~nm}}$ ratio is also dependent on the nature of the ligands protecting the $\mathrm{Au}_{25}$ (for a comparison of $\mathrm{Au}_{25} \mathrm{PMBA}_{18}$ and $\mathrm{Au}_{25}-\mathrm{BSA}$, see Table S1). To better understand the PL properties of the $\mathrm{Au}_{25}$ nanoclusters that attach to $\mathrm{BSA}$ more favorably via cysteine residues, which was suggested by the SAXS/MALDI results, we synthesized mixed liganded nanoclusters, $\mathrm{Au}_{25} \mathrm{pMBA}_{(18-\mathrm{x})} \mathrm{Cys} \mathrm{s}_{\mathrm{x}}$ with $x=2,5,18$, through ligand exchange. Optical measurements and density functional theory allowed us to determine the key role of the metallic-ligand interface in the photophysical pathways.

\section{Insight into the relationship between the structure and optical properties and the origin of the NIR-II} emission. The PL of metal nanoclusters originates from a subtle interplay between excitations arising within the metal core and from charge transfer between the metal core and surface ligands. ${ }^{49,}{ }^{50} \mathrm{~A}$ recent study also suggests the influence of structural distortion accompanied by electron redistribution in photoexcited gold nanoclusters, which induces controllable dual PL emission. ${ }^{51}$ However, the detailed PL mechanism of gold nanoclusters is still under debate. ${ }^{52}$ The high stability and detailed structure determination of $\mathrm{Au}_{25}(\mathrm{SR})_{18}$ (gold nanoclusters protected by SR thiolate ligands) enabled extensive 
investigation of the relationship between the structure and optical properties; in particular, it enabled the study of its PL properties. Upon visible excitation, both red (700-800 nm) and near-infrared emissions (approximately and above $1000 \mathrm{~nm}$ ) have been observed in $\mathrm{Au}_{25}(\mathrm{SR})_{18}$ by different groups. ${ }^{53,54,55} \mathrm{On}$ the basis of time-resolved emission and nanosecond transient absorption spectroscopy analyses, Meng Zhou and Yongbo Song proposed a simplified model, ${ }^{55}$ where visible and near-infrared emissions have different lifetimes and arise from the core-interface charge transfer state and the $\mathrm{Au}_{13}$ core state, respectively.

When $\mathrm{Au}_{25}$ is incorporated into $\mathrm{BSA}$, at least one ligand exchange with the protein occurs via a cysteine residue, which significantly modifies the PL profile (Fig. $3 \mathrm{~b}$ ). To reveal the key role of the metallic-ligand interface in the photophysical pathways of BSA-Au NCs, we produced $\mathrm{Au}_{25} \mathrm{pMBA}_{(18-x)} \mathrm{Cys}_{\mathrm{x}} \mathrm{NCs}$ with $\mathrm{x}=0$, 2,5 , and 18 by ligand exchange (between pMBA and cysteine) from the $A_{25} \mathrm{pMBA}_{18}$ precursor nanocluster. These NCs were fully characterized by ESI-MS (see Fig. S6). $A_{25} \mathrm{PMBA}_{(18-x)} \mathrm{Cys}_{\mathrm{x}}$ with $\mathrm{x}=0$, $<2>,<5>$ and 18 presents the typical absorption features of $\mathrm{Au}_{25} \mathrm{NCs}$ with the characteristic band centered at $680 \mathrm{~nm}$ and a tail band above $780 \mathrm{~nm}$ (Figure 4a). ${ }^{56}$ The PL profile of these $\mathrm{Au}_{25} \mathrm{NCs}$ dispersed in water exhibits two main broad bands at 800-950 nm and 1050-1250 nm (Fig. 4b and c). When the number of Cys molecules on the Au NC surface increased from 2 to 5 , an increase in the PL band at $1050 \mathrm{~nm}$ was observed (see Fig. $4 \mathrm{~b}$ and c). To compare the evolution of the two main NIR-II emission bands, we determined the PL ratio of the peak at $1050 \mathrm{~nm}$ to that at $920 \mathrm{~nm}$ to determine if there is a correlation between the presence of the co-ligand and the PL enhancement at $1050 \mathrm{~nm}$ (Table S1). The results indicated a more pronounced effect when $700 \mathrm{~nm}$ was used as the excitation wavelength than when $400 \mathrm{~nm}$ was used, indicating that the $1050 \mathrm{~nm}$ emission probably involves photophysical relaxation pathways taking place within the metal core. ${ }^{55}$ Indeed, with an excitation wavelength of 700 $\mathrm{nm}$, the lowest energy excited states $(\mathrm{S} 1, \mathrm{~S} 2)$ are mainly involved in photoexcitation, as confirmed by the time-dependent density functional theory (TD-DFT) linear absorption spectrum (Fig. $4 \mathrm{~d}$ and $4 \mathrm{f}$, and see computational details in SI). Low-lying states in the NIR are key to obtaining large two-photon absorption cross sections (due to double resonance effects), which would make them ideal labels for multiphoton excited luminescence (see Fig. S7). The lowest excited states in the absorption spectrum mainly belong to the "core" in Nature, primarily arising from transitions from the occupied P orbitals into the first and second sets of D orbitals (see Fig. $4 \mathrm{e}$ and Table S2). For low-lying excited states, some contributions of the ligands to the transitions are more readily observed in $\mathrm{Au}_{25} \mathrm{pMBA}_{16} \mathrm{Cys}_{2}$ than in $\mathrm{Au}_{25} \mathrm{pMBA}_{18}(\mathrm{Table}$ S2). Under $400 \mathrm{~nm}$ excitation, the nature of excited states is both "interface-like" and "core-like." The characteristic of such excited states is mainly "interface-like" ${ }^{57}$ for which the contribution from the Au-S interface in orbitals is more pronounced, particularly in the cysteine-containing Au NCs (Table S2). Although pMBA and cysteine are rich electron donors, cysteine is more flexible due to the $\mathrm{Ca}-\mathrm{C} \beta$ bond and is better able to interact with the surface of the gold core than pMBA, as its carboxylic group points outward toward the surface of the gold core. Such electron-rich donor groups may contribute to the "interface-like" excited states and thus may increase the contribution of the $850 \mathrm{~nm}$ band. ${ }^{52}$ 
This phenomenon is particularly true for $\mathrm{Au}_{25} \mathrm{Cys}_{18}$, which mainly displays a strong emission at $850 \mathrm{~nm}$. On the basis of these experimental and theoretical results, we propose the following mechanism to explain the relative contribution of emission bands (at 850 and $1050 \mathrm{~nm}$ ) of the $\mathrm{Au}_{25}-\mathrm{BSA}$ conjugates (Figure S8): first, the local environment of $\mathrm{Au}_{25} \mathrm{NCs}$ attached to $\mathrm{BSA}$ is more rigid than free $\mathrm{Au}_{25} \mathrm{pMBA}_{18}$ and prone to multiple energy transfers associated with intersystem crossing (reinforced ISC), which could explain the overall enhancement in PL emission and the longer PL lifetimes. Second, the nature of ligands, and in particular their capability to interact through electron-rich donor groups with the surface of the Au core, may increase the number of surface states involved in excitation (specifically at $400 \mathrm{~nm}$ ).

\section{Discussion}

Here, we present a simple route to attach individual $\mathrm{Au}_{25} \mathrm{NCs}$ to a BSA protein via a cysteine residue. SAXS and molecular modeling provide a clear localization of $\mathrm{Au}_{25}$ within the protein (Cys 34), and MALDI-MS confirms that ligand exchange with a cysteine residue of BSA occurs on the gold nanocluster surface. The change in the local microenvironment of the Au NCs incorporated into the BSA boosts the NIR-II signal but preserves the structure of the $\mathrm{Au}_{25}$. Rigidification of the surface molecules within the BSA protein reinforces the intersystem crossing pathway involved in photoluminescence. Cysteine ligands exchanged during the attachment of $\mathrm{Au}_{25}$ to $\mathrm{BSA}$ allow for an increased contribution of core-interface charge transfer states. Both effects induce enhancement and shift the photoluminescence signal. In addition to the importance of keeping the structure of Au NCs unchanged within the protein, which enables us to decipher the structure-property relationships, it is of interest to use $700 \mathrm{~nm}$ excitation for in vivo optical imaging and photoactivation, both in the linear and nonlinear optical regimes. This study paves the way to designing new photoemitters with tunable NIR-I/NIR-II emission via visible or NIR excitation. Finally, engineering mutations in proteins with cysteines will allow for more selective control of the position of atomically precise NCs in proteins and of the number of ligands exchanged, opening new routes for extending their uses in diagnostic and therapeutic applications.

\section{Declarations}

\section{Acknowledgments}

This research was partly supported by the project STIM-REI, Contract Number: KK.01.1.1.01.0003, funded by the European Union through the European Regional Development Fund - the Operational Programme Competitiveness and Cohesion 2014-2020 (KK.01.1.1.01). VBK, MPB and HF acknowledge computational facilities of the HPC computer within the STIM-REI project, Doctoral study of Biophysics at University of Split as well as Prof. Miroslav Radman at MedILS and Split-Dalmatia County for support. This work was supported by the Labex EpiGenMed, an «Investissements d'avenir» program (ANR-10-LABX-12-01). The CBS is a member of France-Biolmaging (FBI) and the French Infrastructure for Integrated Structural Biology (FRISBI), 2 national infrastructures supported by the French National Research Agency (ANR-10-INBS-04-01 and ANR-10-INBS-05, respectively). We thank the synchrotron 
facility SOLEIL (St. Aubin) for allocating regular beam time (20201085) and its dedicated staff for technical help with the beamline SWING. XLG would like to acknowledge Plan Cancer (C18038CS) and ANR SIREN (ANR-20CE09-0039-01) for their financial support.

\section{Contributions}

F.B., X.LG. and R.A. conceived the idea and designed the experiments. R.A. supervised the project. F.B. C.C.Z. and H.F. synthesised and characterised nanoclusters. K.D.W. and U.T-G carried out SWIR experiments and analysis. P.B. and A.S. carried out SAXS experiments and analysis. M.P.B.. and V.B.K advised and carried out quantum chemistry studies. R.A. wrote the manuscript. All authors analyzed the data, amended and commented on the manuscript.

\section{Corresponding author}

Correspondence to Xavier Le Guével and Rodolphe Antoine.

\section{Ethics declarations}

Competing interests

The authors declare no competing interests.

\section{References}

1. Goswami N, Luo Z, Yuan X, Leong DT, Xie J. Engineering gold-based radiosensitizers for cancer radiotherapy. Materials Horizons 4, 817-831 (2017).

2. Qu X, Li Y, Li L, Wang Y, Liang J, Liang J. Fluorescent Gold Nanoclusters: Synthesis and Recent Biological Application. Journal of Nanomaterials 2015, 23 (2015).

3. Zhang L, Wang E. Metal nanoclusters: New fluorescent probes for sensors and bioimaging. Nano Today 9, 132-157 (2014).

4. Porret E, Le Guével X, Coll J-L. Gold nanoclusters for biomedical applications: toward in vivo studies. Journal of Materials Chemistry B 8, 2216-2232 (2020).

5. Zheng Y, Wu J, Jiang H, Wang X. Gold nanoclusters for theranostic applications. Coordination Chemistry Reviews 431, 213689 (2021).

6. Kurdekar AD, Chunduri LAA, Manohar CS, Haleyurgirisetty MK, Hewlett IK, Venkataramaniah K. Streptavidin-conjugated gold nanoclusters as ultrasensitive fluorescent sensors for early diagnosis of HIV infection. Science Advances 4, eaar6280 (2018).

7. Kang X, Zhu M. Tailoring the photoluminescence of atomically precise nanoclusters. Chemical Society Reviews 48, 2422-2457 (2019).

8. Zhu S, Wang X, Cong Y, Li L. Regulating the Optical Properties of Gold Nanoclusters for Biological Applications. ACS Omega 5, 22702-22707 (2020). 
9. Pyo K, Thanthirige VD, Kwak K, Pandurangan P, Ramakrishna G, Lee D. Ultrabright Luminescence from Gold Nanoclusters: Rigidifying the Au(I)-Thiolate Shell. Journal of the American Chemical Society 137, 8244-8250 (2015).

10. Bertorelle F, et al. Bulky counterions: enhancing the two-photon excited fluorescence of gold nanoclusters. ChemPhysChem, 19, 165-168 (2018).

11. Le Guével $X$, Tagit $O$, Rodríguez CE, Trouillet V, Pernia Leal M, Hildebrandt N. Ligand effect on the size, valence state and red/near infrared photoluminescence of bidentate thiol gold nanoclusters.

Nanoscale 6, 8091-8099 (2014).

12. Chen $Y$, et al. Shortwave Infrared in Vivo Imaging with Gold Nanoclusters. Nanoletters 17, 6330-6334 (2017).

13. Perić $M$, et al. Ligand shell size effects on one- and two-photon excitation fluorescence of zwitterion functionalized gold nanoclusters. Physical Chemistry Chemical Physics 21, 23916-23921 (2019).

14. Zhang B, Chen J, Cao Y, Chai OJH, Xie J. Ligand Design in Ligand-Protected Gold Nanoclusters. Small 17, 2004381 (2021).

15. Crawford SE, Hartmann MJ, Millstone JE. Surface Chemistry-Mediated Near-Infrared Emission of Small Coinage Metal Nanoparticles. Accounts of Chemical Research 52, 695-703 (2019).

16. Crawford SE, et al. Ligand-Mediated "Turn On," High Quantum Yield Near-Infrared Emission in Small Gold Nanoparticles. Journal of the American Chemical Society 137, 14423-14429 (2015).

17. Andolina CM, Dewar AC, Smith AM, Marbella LE, Hartmann MJ, Millstone JE. Photoluminescent Gold-Copper Nanoparticle Alloys with Composition-Tunable Near-Infrared Emission. Journal of the American Chemical Society 135, 5266-5269 (2013).

18. Musnier B, et al. High photoluminescence of shortwave infrared-emitting anisotropic surface charged gold nanoclusters. Nanoscale 11, 12092-12096 (2019).

19. Yu Z, et al. High-Resolution Shortwave Infrared Imaging of Vascular Disorders Using Gold Nanoclusters. ACS Nano 14, 4973-4981 (2020).

20. Zare I, et al. Protein-protected metal nanoclusters as diagnostic and therapeutic platforms for biomedical applications. Materials Today, (2021).

21. Xie J, Zheng Y, Ying JY. Protein-Directed Synthesis of Highly Fluorescent Gold Nanoclusters. Journal of the American Chemical Society 131, 888-889 (2009).

22. Shang L, Dong S, Nienhaus GU. Ultra-small fluorescent metal nanoclusters: synthesis and biological applications. Nano Today 6, 401-418 (2011).

23. Shang L, Nienhaus GU. Gold nanoclusters as novel optical probes for in vitro and in vivo fluorescence imaging. Biophysical Reviews 4, 313-322 (2012).

24. Shang L, Xu J, Nienhaus GU. Recent advances in synthesizing metal nanocluster-based nanocomposites for application in sensing, imaging and catalysis. Nano Today 28, 100767 (2019).

25. Liu C-L, et al. Insulin-Directed Synthesis of Fluorescent Gold Nanoclusters: Preservation of Insulin Bioactivity and Versatility in Cell Imaging. Angewandte Chemie International Edition 50, 7056-7060 
(2011).

26. Zhuang Q-Q, et al. Immunoglobulin G-Encapsulated Gold Nanoclusters as Fluorescent Tags for DotBlot Immunoassays. ACS Applied Materials \& Interfaces 11, 31729-31734 (2019).

27. Zhuang Q-Q, et al. Detection of tetanus toxoid with fluorescent tetanus human IgG-AuNC-based immunochromatography test strip. Biosensors and Bioelectronics 177, 112977 (2021).

28. Soleilhac A, Bertorelle F, Antoine R. Sizing protein-templated gold nanoclusters by time resolved fluorescence anisotropy decay measurements. Spectrochimica Acta Part A: Molecular and Biomolecular Spectroscopy 193, 283-288 (2018).

29. Baksi A, Mitra A, Mohanty JS, Lee H, De G, Pradeep T. Size Evolution of Protein-Protected Gold Clusters in Solution: A Combined SAXS-MS Investigation. The Journal of Physical Chemistry C119, 2148-2157 (2015).

30. Ghosh D, et al. Gold-Induced Unfolding of Lysozyme: Toward the Formation of Luminescent Clusters. The Journal of Physical Chemistry C 121, 13335-13344 (2017).

31. Chaudhari K, Xavier PL, Pradeep T. Understanding the Evolution of Luminescent Gold Quantum Clusters in Protein Templates. ACS Nano 5, 8816-8827 (2011).

32. Kluz M, et al. Revisiting the conformational state of albumin conjugated to gold nanoclusters: A selfassembly pathway to giant superstructures unraveled. PLOS ONE 14, e0218975 (2019).

33. Chevrier DM, et al. Structure and formation of highly luminescent protein-stabilized gold clusters. Chemical Science 9, 2782-2790 (2018).

34. Kindi HA, et al. Single bovine serum albumin molecule can hold plural blue-emissive gold nanoclusters: A quantitative study with two-photon excitation. Journal of Photochemistry and Photobiology A: Chemistry 357, 168-174 (2018).

35. Ghosh D, et al. Internalization of a Preformed Atomically Precise Silver Cluster in Proteins by Multistep Events and Emergence of Luminescent Counterparts Retaining Bioactivity. The Journal of Physical Chemistry C123, 29408-29417 (2019).

36. Wang Y, Bürgi T. Ligand exchange reactions on thiolate-protected gold nanoclusters. Nanoscale Advances 3, 2710-2727 (2021).

37. Combes GF, et al. Functionalized Au15 nanoclusters as luminescent probes for protein carbonylation detection. Communications Chemistry 4, 69 (2021).

38. Combes GF, Vučković A-M, Perić Bakulić M, Antoine R, Bonačić-Koutecky V, Trajković K. Nanotechnology in Tumor Biomarker Detection: The Potential of Liganded Nanoclusters as Nonlinear Optical Contrast Agents for Molecular Diagnostics of Cancer. Cancers 13, 4206 (2021).

39. Bertorelle F, et al. Isomeric Effect of Mercaptobenzoic Acids on the Synthesis, Stability, and Optical Properties of Au25(MBA) 18 Nanoclusters. ACS Omega 3, 15635-15642 (2018).

40. Koch MHJ, Vachette P, Svergun DI. Small-angle scattering: a view on the properties, structures and structural changes of biological macromolecules in solution. Quarterly Reviews of Biophysics 36, 147-227 (2003). 
41. Putnam CD, Hammel M, Hura GL, Tainer JA. X-ray solution scattering (SAXS) combined with crystallography and computation: defining accurate macromolecular structures, conformations and assemblies in solution. Q Rev Biophys 40, 191-285 (2007).

42. Bernadó P, Shimizu N, Zaccai G, Kamikubo H, Sugiyama M. Solution scattering approaches to dynamical ordering in biomolecular systems. Biochimica et Biophysica Acta (BBA) - General Subjects 1862, 253-274 (2018).

43. Jeffries CM, Graewert MA, Blanchet CE, Langley DB, Whitten AE, Svergun DI. Preparing monodisperse macromolecular samples for successful biological small-angle X-ray and neutron-scattering experiments. Nature Protocols 11, 2122-2153 (2016).

44. Panjkovich A, Svergun DI. Deciphering conformational transitions of proteins by small angle X-ray scattering and normal mode analysis. Physical Chemistry Chemical Physics 18, 5707-5719 (2016).

45. Kang X, Chong H, Zhu M. Au25(SR)18: the captain of the great nanocluster ship. Nanoscale 10, 10758-10834 (2018).

46. Zhu M, Aikens CM, Hollander FJ, Schatz GC, Jin R. Correlating the Crystal Structure of A ThiolProtected Au25 Cluster and Optical Properties. Journal of the American Chemical Society 130, 58835885 (2008).

47. Yu H, Rao B, Jiang W, Yang S, Zhu M. The photoluminescent metal nanoclusters with atomic precision. Coordination Chemistry Reviews 378, 595-617 (2019).

48. Huang Y, Fuksman L, Zheng J. Luminescence mechanisms of ultrasmall gold nanoparticles. Dalton Transactions 47, 6267-6273 (2018).

49. Weerawardene KLDM, Aikens CM. Theoretical Insights into the Origin of Photoluminescence of Au25(SR)18- Nanoparticles. Journal of the American Chemical Society 138, 11202-11210 (2016).

50. Yang T-Q, et al. Origin of the Photoluminescence of Metal Nanoclusters: From Metal-Centered Emission to Ligand-Centered Emission. Nanomaterials 10, 261 (2020).

51. Li Q, et al. Structural distortion and electron redistribution in dual-emitting gold nanoclusters. Nature Communications 11, 2897 (2020).

52. Zhou M, Jin R. Optical Properties and Excited-State Dynamics of Atomically Precise Gold Nanoclusters. Annual Review of Physical Chemistry 72, 121-142 (2021).

53. Link S, Beeby A, FitzGerald S, El-Sayed MA, Schaaff TG, Whetten RL. Visible to Infrared Luminescence from a 28-Atom Gold Cluster. The Journal of Physical Chemistry B 106, 3410-3415 (2002).

54. Wu Z, Jin R. On the Ligand's Role in the Fluorescence of Gold Nanoclusters. Nano Letters 10, 25682573 (2010).

55. Zhou M, Song Y. Origins of Visible and Near-Infrared Emissions in [Au25(SR)18]- Nanoclusters. The Journal of Physical Chemistry Letters 12, 1514-1519 (2021).

56. Zhao S, et al. Influence of Atomic-Level Morphology on Catalysis: The Case of Sphere and Rod-Like Gold Nanoclusters for CO2 Electroreduction. ACS Catalysis 8, 4996- 5001 (2018). 
57. Aikens CM. Electronic and Geometric Structure, Optical Properties, and Excited State Behavior in Atomically Precise Thiolate-Stabilized Noble Metal Nanoclusters. Accounts of Chemical Research 51, 3065-3073 (2018).

\section{Figures}

Step I : formation of $\mathrm{Au}_{25} \mathrm{pMBA}_{18}$
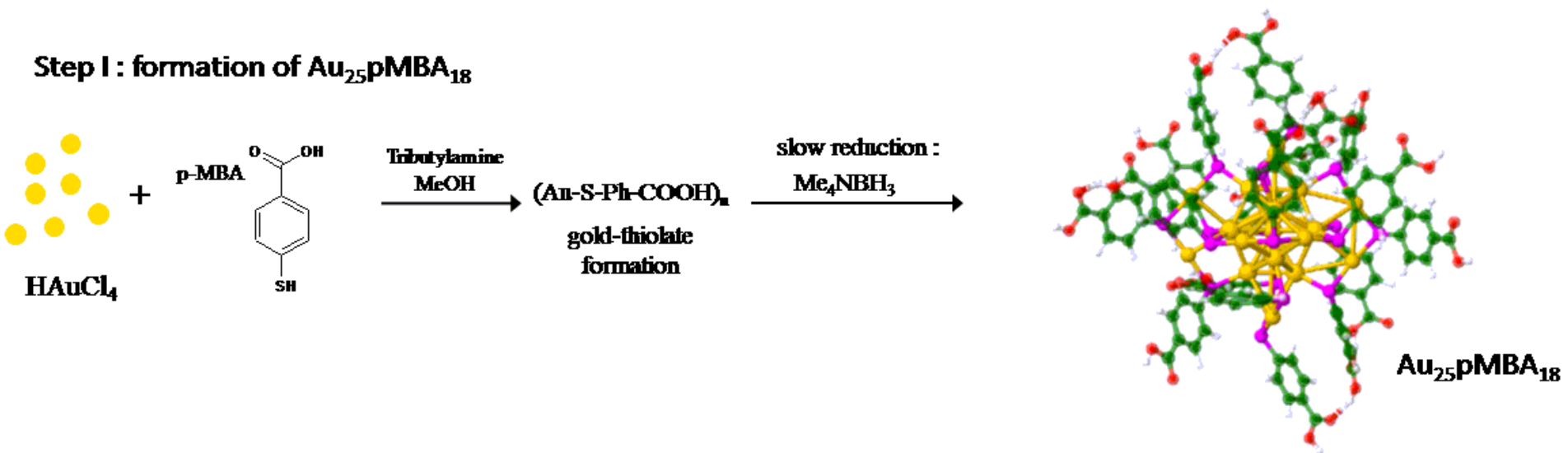

Step II : incorporation of $\mathrm{Au}_{25}$ within BSA template via ligand exchange reaction
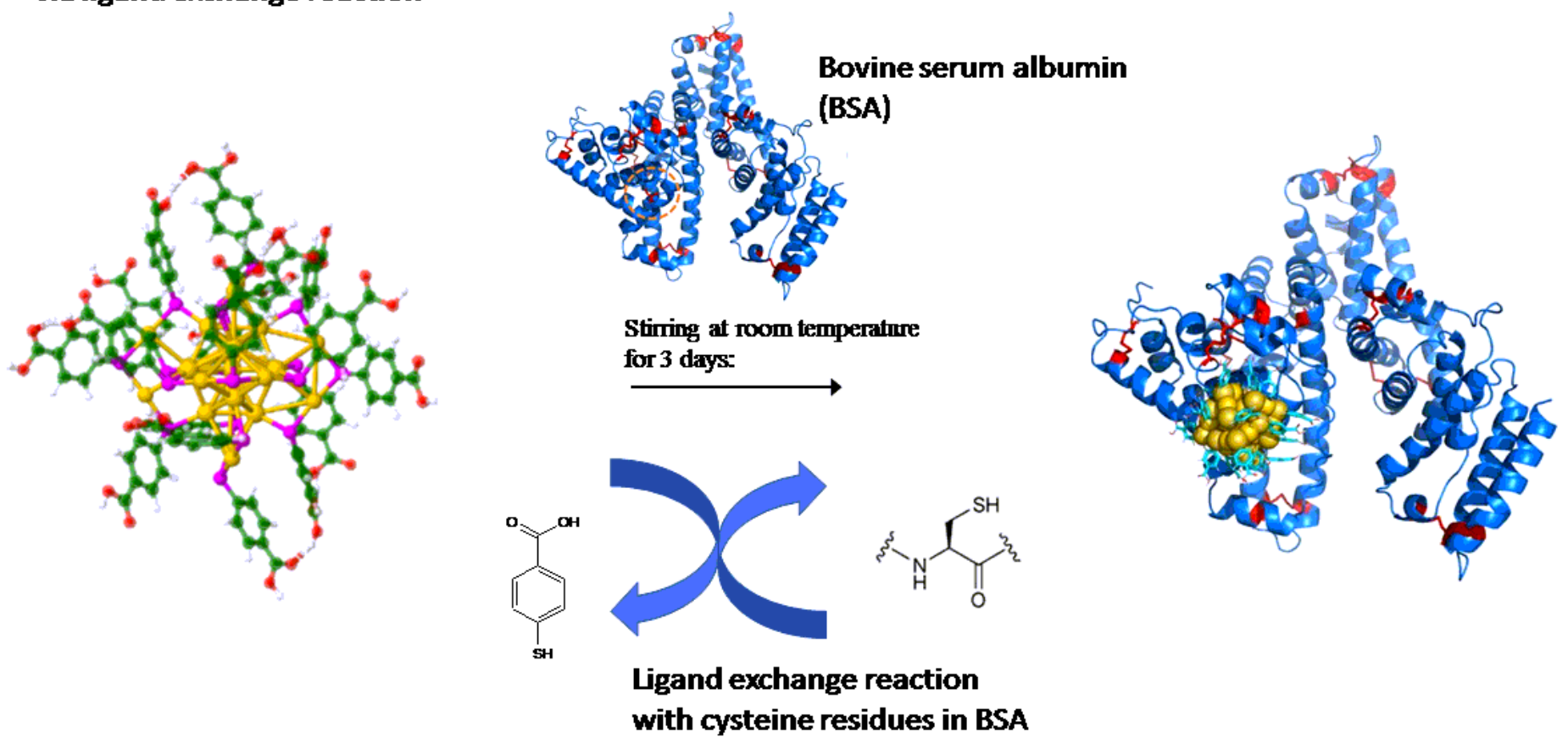

Figure 1

Schematic illustration of the reaction process leading from the formation of Au25 nanoclusters to their attachment to BSA. Step I. Formation of thiolated Au25 NCs. Step II. Ligand exchange with cysteine residues in the BSA leading to the anchoring of Au25 nanoclusters to BSA. Cysteines are highlighted in red, and the Au25 NC structure is shown anchored to Cys34. 


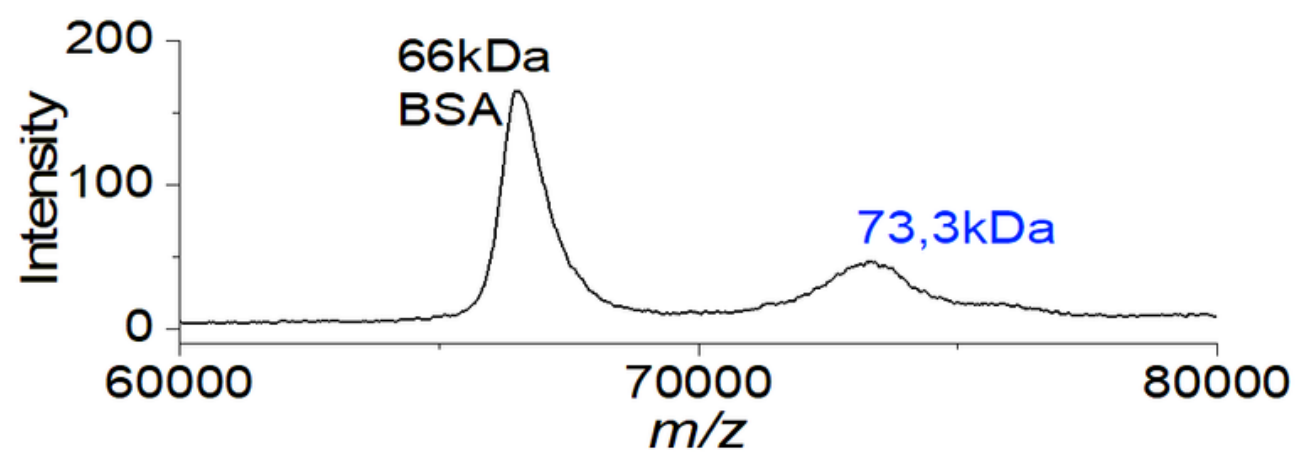

b
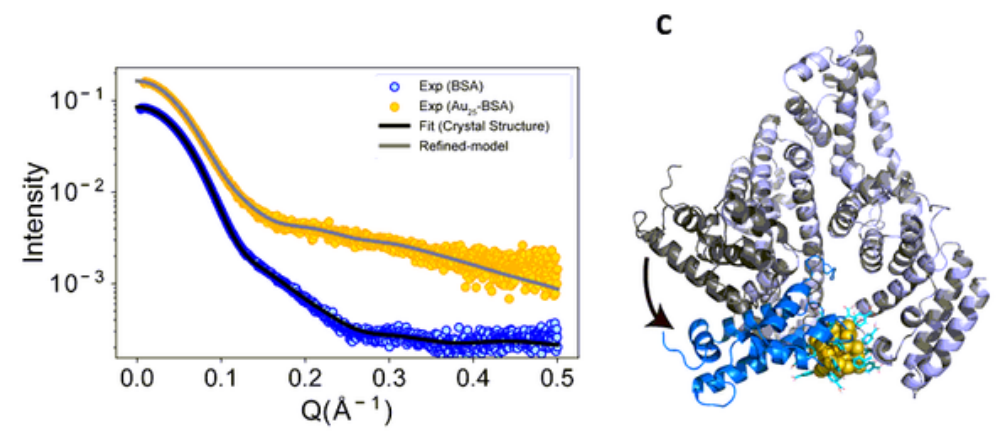

d
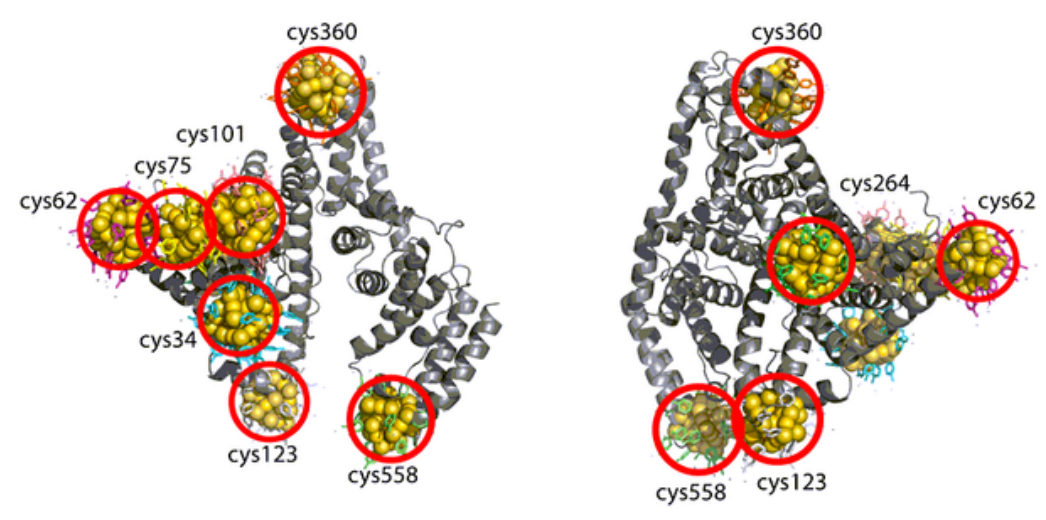

e

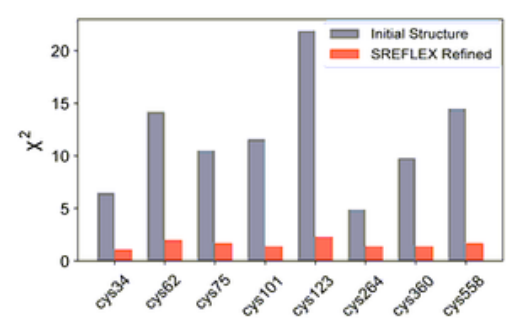

Figure 2

a) MALDI-TOF spectrum of Au25 nanoclusters attached to BSA protein (positive mode). b) The experimental SAXS profiles of BSA (blue) overlaid with the theoretical SAXS profile of BSA monomer calculated using Crysol (black) and the SAXS profile of Au25-BSA (orange) overlaid with the theoretical SAXS profile of the SREFLEX refined model with Au25 bound to Cys34. c) The SREFLEX refined model of the Au25-BSA (light blue) superimposed on the crystal structure of BSA. The position of the part that was 
allowed to be moved by SREFLEX is shown in darker shades of the same colors in both structures. d) Two views of the BSA conjugated to Au25 at various cysteines, tested to determine the agreement with the SAXS data. e) The $\chi 2$ values between the theoretical SAXS profile of BSA conjugated to Au25 at different cysteines calculated directly from the structure using Crysol (gray bars) or after refinement using SREFLEX (red bars).

a

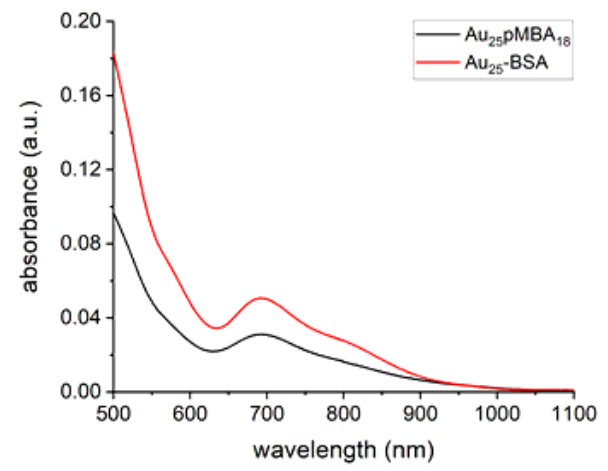

b

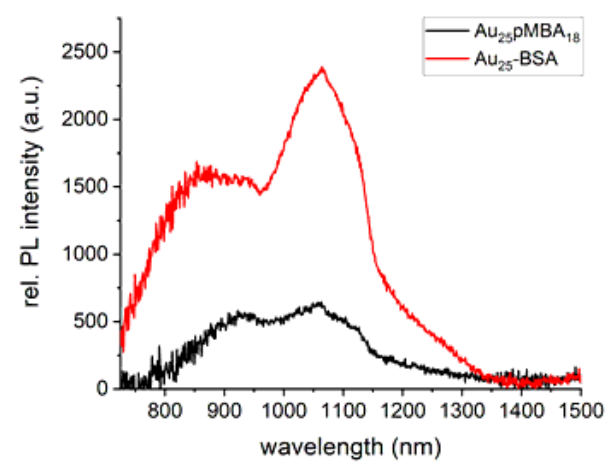

d

\begin{tabular}{|c|c|c|c|c|}
\hline$\lambda_{a x c}=700 \mathrm{~nm}$ & $700-1500$ & $\begin{array}{c}\text { n (refractive } \\
\text { index) }\end{array}$ & $\mathbf{Q Y}$ & $\mathbf{Q Y}(\%)$ \\
\hline IR125 & $2.12 E+07$ & 1.48 & 0.23 & \\
\hline $\mathrm{Au}_{35} \mathrm{pMBA}$ & $1.76 E+05$ & 1.33 & 0.002 & 0.15 \\
\hline $\mathrm{Au}_{55} \mathrm{pMBA}_{16} \mathrm{Cys}_{2}$ & 2.27E+05 & 1.33 & 0.002 & 0.20 \\
\hline $\mathrm{Au}_{25} \mathrm{pMBA}_{13} \mathrm{CyS}_{5}$ & $3.23 E+05$ & 1.33 & 0.003 & 0.28 \\
\hline $\mathrm{Au}_{35} \mathrm{Cys}_{13}$ & $3.73 E+05$ & 1.33 & 0.003 & 0.33 \\
\hline $\mathrm{Au}_{35}-\mathrm{BSA}$ & 1,11E+06 & 1.33 & 0.007 & 0.70 \\
\hline
\end{tabular}

c
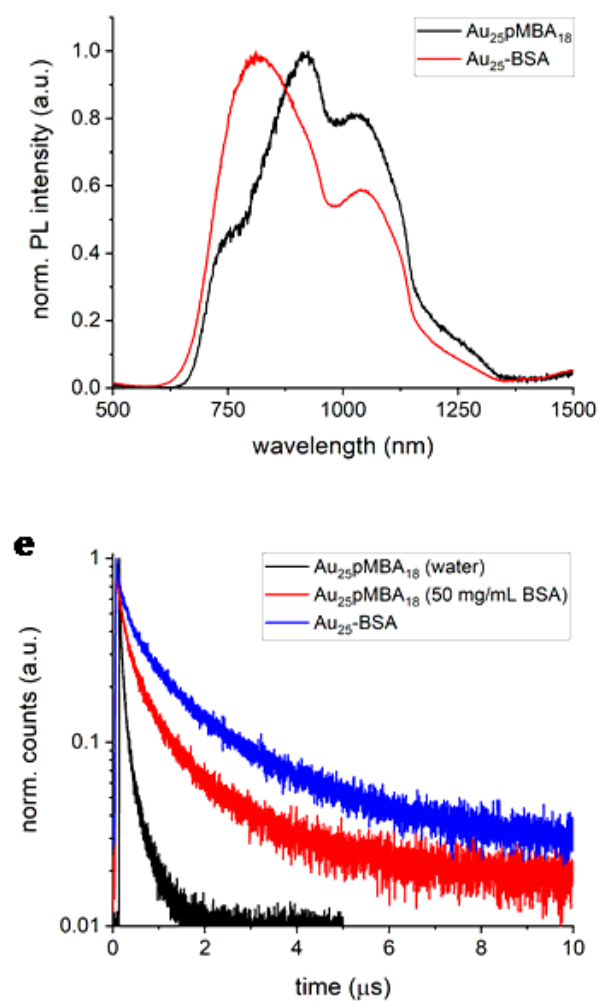

Figure 3

a) Absorption spectra of Au25pMBA18 and BSA-Au25 in water. PL spectra of Au25pMBA18 and BSAAu25 in water, with (b) $\lambda$ exc. $700 \mathrm{~nm} \mathrm{~b}$ ) and c) $\lambda$ exc. d) Table 1: Relative QY of Au25pMBA(18-x)Cys-x and Au25-BSA at $700 \mathrm{~nm}$ excitation using IR125 as a reference. e) PL lifetime decay of Au25pMBA18 in water, Au25pMBA18 with BSA, and BSA-Au25 in water ( $\lambda$ exc. $485 \mathrm{~nm}$; $\lambda$ em. $920 \mathrm{~nm}$ ). 
a

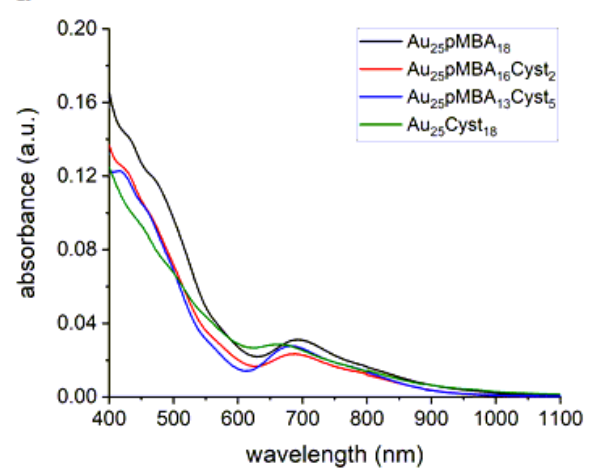

b

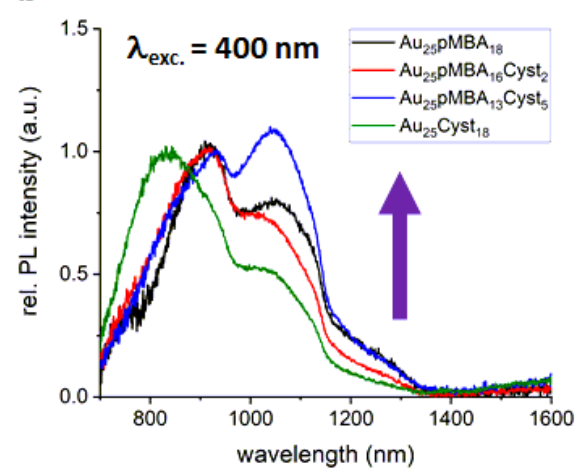

C

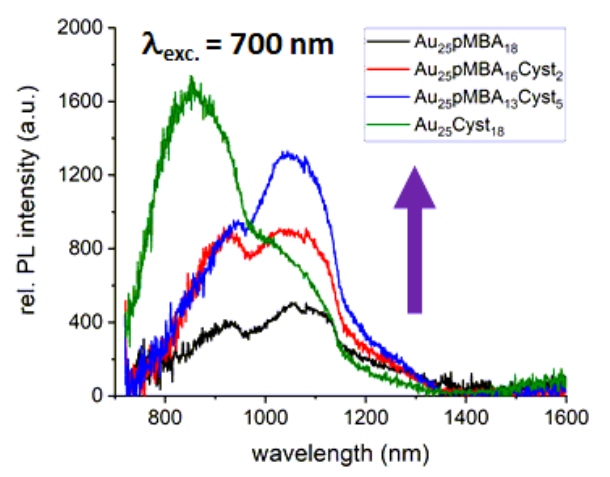

d

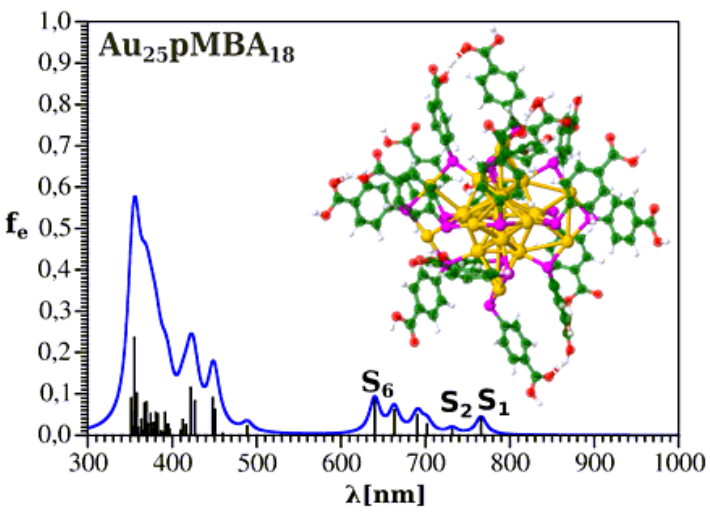

f

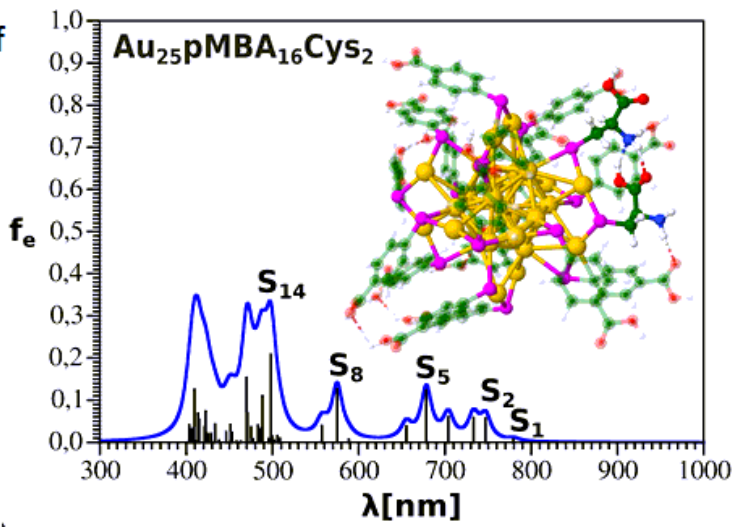

e

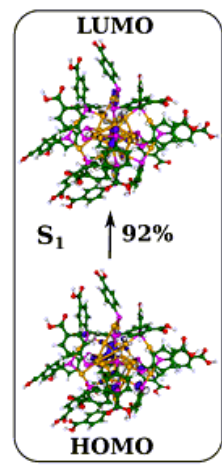

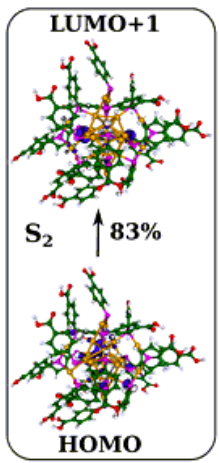

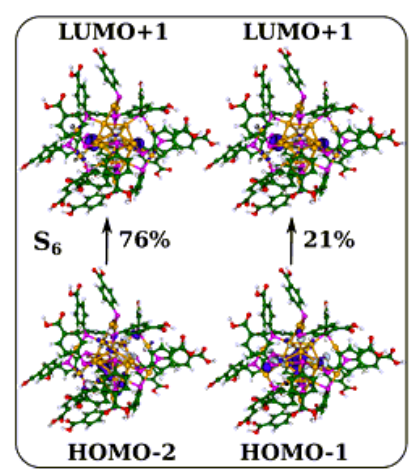

g
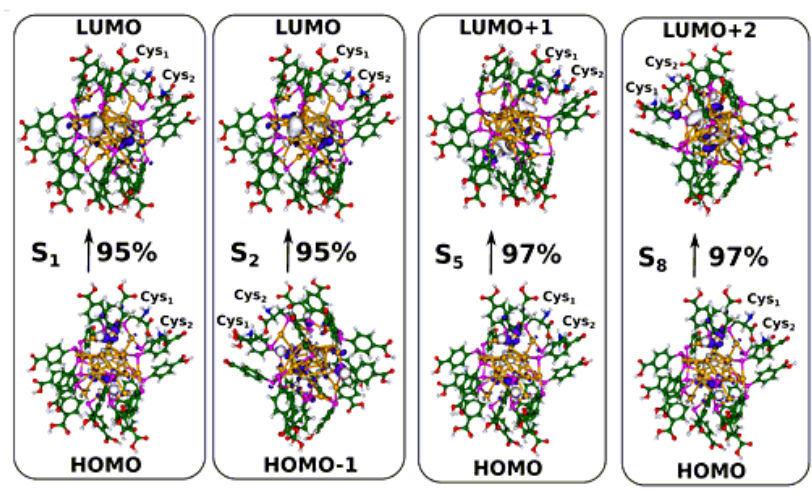

\section{Figure 4}

(a) Absorbance spectra and (b,c) normalized PL spectra of Au25pMBA(18-x)Cysx solutions dispersed in water. d) and e) TD-DFT results on Au25pMBA18: absorption spectrum and molecular orbitals involved in the first excited state transitions, showing involvement of the gold core and contributions from Au-S, Au or S. f) and g) TD-DFT results on the Au25pMBA16Cys2 absorption spectrum and molecular orbitals involved in the first excited state transitions, showing the involvement of the gold core and contributions from Au-S, Au or S.

\section{Supplementary Files}

This is a list of supplementary files associated with this preprint. Click to download. 
- Supportinginformation051021.docx 